This item was submitted to Loughborough's Research Repository by the author.

Items in Figshare are protected by copyright, with all rights reserved, unless otherwise indicated.

\title{
Dipolar clusters and ferroelectricity in high Tc superconductors
}

PLEASE CITE THE PUBLISHED VERSION

http://dx.doi.org/10.1142/S0217979215420023

\section{PUBLISHER}

(c) World Scientific Publishing Company

\section{VERSION}

AM (Accepted Manuscript)

\section{PUBLISHER STATEMENT}

This work is made available according to the conditions of the Creative Commons Attribution-NonCommercialNoDerivatives 4.0 International (CC BY-NC-ND 4.0) licence. Full details of this licence are available at: https://creativecommons.org/licenses/by-nc-nd/4.0/

\section{LICENCE}

CC BY-NC-ND 4.0

\section{REPOSITORY RECORD}

Kusmartsev, F.V., and M. Saarela. 2019. "Dipolar Clusters and Ferroelectricity in High Tc Superconductors". figshare. https://hdl.handle.net/2134/18829. 


\title{
DIPOLAR CLUSTERS AND FERROELECTRICITY IN HIGH Tc SUPERCONDUCTORS *
}

\author{
F. V. KUSMARTSEV ${ }^{\dagger}$ \\ Department of Physics, Loughborough University \\ Loughborough, LE11 $3 T U, U K \ddagger$ \\ F.Kusmartsev@lboro.ac.uk $\S$ \\ M. SAARELA \\ Department of Physical Sciences, University of Oulu \\ P.O.Box 3000, FIN-90014 University of Oulu, Finland \\ Mikko.Saarela@oulu.fi
}

\begin{abstract}
We show that doping of hole charge carriers induces formation of resonance plaquettes(RP) having electric dipolar moments and fluctuating stripes in cuprates. A single $\mathrm{RP}$ is created by many-body interactions between the dopant ion or a charge fluctuation outside and holes inside the $\mathrm{CuO}$ plane. In such a process Coulomb interacting holes in the $\mathrm{CuO}$ plane are self-organised into 4-particles resonance valence bond plaquettes bound with dopants or polarons located in the spacer layer between $\mathrm{CuO}$ planes. Such RPs have ordered and disordered phases. They are ordered into charge-density waves (CDW) or stripes only at certain conditions. The lowest energy of the ordered phase corresponds to a local anti-ferroelectric ordering. The RPs mobility is very low at low temperatures and they are bound into dipole-dipole pairs. Electromagnetic radiation interacts strongly with RPs electric dipoles and when the sample is subjected to it the mobility changes significantly. This leads to a fractal growth of dipolar RP clusters. The existence of electric dipoles and CDW reveal a series of new phenomena such as ferroelectricity, strong light and microwave absorption and the field induced superconductivity.
\end{abstract}

Keywords: Charge density wave; anti-ferroelectricity; Jahn-Teller effect, pseudogap and high-temperature superconductivity, two-dimensional Luttinger liquid

A guided migration of doped oxygen atoms in $\mathrm{La}_{2} \mathbf{C u O}_{4+y}$ with nano-scale synchrotron radiation scanning X-ray diffraction in the temperature range $180 \mathrm{~K}$ $<\mathrm{T}<330 \mathrm{~K}$ found in Refs ${ }^{1,2}$ created a huge puzzle on its origin. Randomly distributed Oxygen dopants the LaO plane order into clusters after of illumination. The higher superconducting transition temperature arises with their better order. These fundamental discoveries ${ }^{1,2}$ pose the questions: Why the radiation increases the mobility of oxygen ions?, Why they are ordered? and Why

*DIPOLAR CLUSTERS AND FERROELECTRICITY IN HIGH Tc SUPERCONDUCTORS

${ }^{\dagger}$ Department of Physics, Loughborough University, LE11 3TU, UK

¥Institute of Quantum Technologies, Loughborough, LE11 3TU, UK

§F.Kusmartsev@lboro.ac.uk 
the superconductivity arises at higher temperature with this order?. The inhomogeneity of cuprate superconductors coupled with dopant atoms is well documented also in other materials and in different experiments like using scanning tunneling microscope ${ }^{3,4,5,6}$, photoemission spectra (ARPES) ${ }^{7}$ suggesting that the oxygen dopant-induced states are mixed with $\mathrm{Cu}$ atoms and measurements of dielectric constant etc. ${ }^{8}$ So, In $\mathrm{Sr}$ doped $\mathrm{La}_{2-x} \mathrm{Sr}_{x} \mathrm{CuO}_{4}$ (LSCO) ${ }^{9,10}$ a peculiar temperature dependence of the Hall coefficient, $\mathrm{R}_{H}$, has been observed and analyzed within a two-band model. It was argued that the effective hole density can be written as the sum of two components $n_{\mathrm{eff}}(x, T)=x+n_{1} e^{-\Delta(x) / 2 k_{B} T}$. The analysis suggested that at small doping , $0.01<x<0.08$, and below the room temperature each dopant atom creates one hole. At higher temperatures the hole density increases rapidly and for each $x$ that temperature behavior of the density was well fitted with an activation law. The true nature of this two-band behaviour and the activation energy $\Delta(x)$ was left as an open question ${ }^{10}$. At low doping the coefficient $n_{1}$ fitted to the experimental data is independent of $x$ and larger than 1 hole/Cu. In Ref. [9] the value $n_{1}=2.8$ and in Ref. [10] the value $n_{1} \approx 4$ was found. At higher doping $0.08<x<0.21$ and below the room temperature the hole density increases as a function of doping faster than the number of dopant atoms and, more strangely, for a given $x$ when the temperature increases from $0 \mathrm{~K}$ to $50 \mathrm{~K}$ the carrier density decreases. This suggests that the charge density can fluctuate between the two bands. Therefore, LSCO is not behaving as a simple, single-band, but rather a two-band correlated non-Fermi liquid ${ }^{11,12}$.

Debate between two competing orders in the pseudogap region focuses mainly in the interpretation of ARPES measurements on the nature of gap functions and Fermi arcs. ${ }^{13,14,15,16,17}$ Very recent experiments found evidences of sharp phase transitions at temperatures between the superconducting critical temperature $\mathrm{T}_{c}$ and the pseudogap temperature at $\mathrm{T}^{*} .{ }^{18,19}$ At zero temperature inside the superconducting dome there are two phase transitions ${ }^{20}$. Also the temperature dependence of resistivity changes from quadratic to linear behavior inside the pseudogap region with increasing doping and temperature. ${ }^{21,22}$

Using the microscopic approach ${ }^{23}$ we show that here bound states appear because dopant atoms e. g. $\mathrm{Sr}^{2+}$ are replacing the $\mathrm{La}^{3+}$, and therewith inducing extra negative charges into spacer layers. At low doping these negative charges are able to bind holes. The key ingredient in binding holes into dopant atom is the near degeneracy of the d-state bands with $x^{2}-y^{2}$ and $z^{2}$ symmetry in the $\mathrm{CuO}$ plane. The Jahn-Teller distortions in cuprates, such as $\mathrm{La}_{2} \mathrm{CuO}_{4}$, elongate the oxygen octahedron surrounding the central $\mathrm{Cu}$ ion and split the degeneracy of the associated $e_{g}$ orbitals of the $\mathrm{Cu} d^{9}$-state. This happens in the highest partially occupied $x^{2}-y^{2}$ orbital, which together with the doubly occupied $\mathrm{O} p_{x}, p_{y}$-orbitals form a strong covalent bonding. Their straight hybridization gives rise to the bonding, non-bonding and half-filled anti-bonding bands and predicts a good metal, in sharp contrast with experiments finding a large charge gap. The failure of the band theory indicates a strong Coulomb interaction (e.g. on site Hubbard $U$ ), which may exceed well the 
bandwidth of the tight-binding, anti-bonding band.

The Hubbard $U$ is much larger than the energy separation between the $\mathrm{Cu}$ $x^{2}-y^{2}$ and $\mathrm{O} p$-orbitals-the charge transfer gap $\Delta_{C T} \sim 1-2 \mathrm{eV}$, which is much larger than the hybridisation integral, $t_{d p} \sim 0.350 \mathrm{meV}^{24,25,26}$. Therefore single electrons are localized on $\mathrm{Cu}$ sites on the $\mathrm{Cu} x^{2}-y^{2}$ orbitals forming the $\mathrm{Cu}^{2+}$ states. Their spins are anti-ferromagnetically aligned (via the super-exchange interaction that involves virtual hopping to the neighbouring $\mathrm{O} p$ - orbitals) to create the anti-ferromagnetic Mott insulator ${ }^{27}$. The formal valence state $\mathrm{Cu}^{3+}$, which should arise with doping would already have large negative $\Delta_{C T}<0$ and therefore should be rather represented by Zhang-Rice singlet, i.e. as $\mathrm{Cu}^{3+} \rightarrow \mathrm{Cu}^{2+} L$, (see, the Ref. ${ }^{28}$ for a detail). There the doped holes would go not so much to the d-shells but rather to the oxygens, creating oxygen holes (though the quantum numbers of the respective states would be the same as those of $\mathrm{Cu}^{3+}$ ). In these Zhang-Rice singlets the hole is distributed between the $\mathrm{Cu}^{3+}$ and four neighbouring oxygens. Cuprates, like $\mathrm{La}_{2} \mathrm{CuO}_{4}$, are layered ionic crystals. The $\left(\mathrm{CuO}_{2}\right)^{2-}$ layer is negatively charged and two spacer layers $\left(\mathrm{La}_{2} \mathrm{O}_{2}\right)^{2+}$ are positively charged. Oxygen atoms form an octahedral cell around $\mathrm{Cu}^{2+}$ and the apex oxygen atoms containing two extra electrons filling the p-shell are in the LaO- layer. In the parent anti-ferromagnetic insulator compound the degeneracy of cubic $e_{g}$ states is removed by the Jahn-Teller effect, which elongates the oxygens octahedron. Copper, on the other hand, is a transition metal and it can easily give out one electron and transfer into $\mathrm{Cu}^{3+} \rightarrow \mathrm{Cu}^{2+} L$ sharing the electron with octahedron oxygens and forming Zhang-Rice singlet.

Dopant atoms settle in the $\mathrm{LaO}$ spacer layer substituting $\mathrm{La}$ atoms like in the case of $\mathrm{Sr}$ doping or intercalated like oxygen atoms. Oxygen atoms are small in size and thus mobile. They easily fill their p-shell with two electrons and become ionized. When this happens the negative impurity charge pushes the negatively charged apex oxygen down to $\mathrm{CuO}$ plane. This restores the $e_{g}$ symmetry and helps to remove one electron from neighbouring $\mathrm{Cu}$-ions to form Zhang-Rice singlet (the anti-Jahn-Teller effect). ${ }^{a}$ At large enough doping holes created ("Zhang-Rice singlet") band in the $\mathrm{CuO}$ plane. Many complexes containing specific transition-metal central ions with special valency show this effect. ${ }^{29}$ Every new hole appearing during hole-doping in the parent compound leads to strong frustration of the original antiferromagnetic state. This leads to rapid suppression of antiferromagnetism in $\mathrm{La}_{2-x} \mathrm{Sr}_{x} \mathrm{CuO}_{4}$ even by small hole concentration. Four holes bound by dopant atoms due to correlations ${ }^{30}$ form a resonance plaquette(RP). It is similar to the resonance valence bonds described by Anderson ${ }^{27}$. These RPs together with dopant atoms or polarons induce electrical dipolar moments.

The interaction between dipolar RPs is short-ranged and varies as a function of separation distance with the $1 / r^{3}$ tail. Because of their low mobility, CDW nucleation or RPs condensation starts at different parts of the sample and with dop-

\footnotetext{
a Note that with this anti-Jahn-Teller effect associated with the squeezing of the oxygen octahedra, the hole state has a two fold degeneracy that is in addition of the conventional Kramers degeneracy.
} 
ing lead to patches of different directional order with a fractal structure There are strings or stripes like those which arise due to electron-phonon interaction ${ }^{31,32,33}$. Any isolated charge associated with an impurity or a charge fluctuation in $\mathrm{LaO}$ spacer is inducing the anti-Jahn-Teller shift of the apex $\mathrm{O}$ ion ${ }^{23,30}$ and therewith creates $\mathrm{RP}$ in $\mathrm{CuO}$ plane. To describe these RPs we developed the many-body variational theory where four holes are trapped by the impurity. ${ }^{23,34,35,36}$. and derived a proper energetic description of RP states which reproduce experimental pseudogap results ${ }^{9,10}$. Here we assume that the hole band is two-fold degenerate. The RPs behave as quasi-classical objects moving along in a nearly flat band and form a two- dimensional Luttinger liquid described in the Ref. ${ }^{37}$ or orbital Non-Fermi liquid $^{12}$ or glass ${ }^{38}$. The RP fluctuations play a huge role in the scattering of the remaining mobile holes and are a source of Planckian dissipations, which has the shortest possible relaxation time named as the Planck time ${ }^{39}$.

An irradiation create excitons of intermediate radius around RP. Such an exciton may create a pair of lattice defects ${ }^{40}$ such as the negatively charged oxygen interstitials, i-O, and the positively charged vacancy, $\mathrm{V}+$. Such excitonic mechanism of the defect production has been observed experimentally ${ }^{41,42}$. When the vacancy, $\mathrm{V}+$ will approach to another i-O, they annihilate. The creation of such virtual pairs increases significantly the mobility of the i-O's and stripy local lattice distortions, noted as Q3 are created ${ }^{43,44}$. The probability to such excitons with X-ray illumination very fast vanishes with the doping. This explains why the Q3 clusters are arising only in the very underdoped cuprates.

The density of dipole moments associated with RPs increases with doping and vanishes at the pseudogap temperature. The binding energy of holes into these dipolar clusters defines the pseudogap, which we identify also as the activation energy of the two fluid model used by Gorkov and Teitelbaum. ${ }^{9,10,45}$ Dipole moments have a strong interaction with electromagnetic radiation and subjected to such radiation dipoles become mobile. Due to the dipole-dipole attraction existing for some dipole orientations and repulsion for other orientations they may form clusters. This finding explains the recent observation of the self-organization of mobile oxygen dopant ions in LCO by Bianconi et al. ${ }^{1,2}$. Under irradiation oxygen ions resting in the $\mathrm{LaO}$ layer as well as dipoles get excited and are forced to move towards the energetically most favourable positions. Ferroelectricity in $\mathbf{L a}_{2} \mathbf{C u O} \mathbf{O}_{4+y}$ was reported at exceptionally low oxygen doping. ${ }^{46}$ To explain this the magneto-electric coupling was used ${ }^{47}$. The ferroelectricity may arise due to electric dipoles. We think that the dipole formation is the source of the ferroelectricity seen in recent experiments ${ }^{46}$ and of the peculiar behaviour of the dielectric constant ${ }^{8}$. The RPs described may also play a very important role in the mechanism of high temperature superconductivity. Their presence in the vicinity of the hole band edge may lead to shape resonances as described recently by Bianconi ${ }^{48}$, that can be an origin of the Cooper pairing in cuprates. Our results suggest that pseudogap formation and high temperature superconductivity are interaction driven and therefore these materials belong to a new class of holographic superconductors ${ }^{49}$. 
1. M. Fratini,et al, Nature 466, p. 841 (2010).

2. N. Poccia, et al, Nature Materials 10, p. 733 (2011).

3. P. Mallet, et al, Phys. Rev. B 54, 13324(Nov 1996).

4. T. Cren, D. Roditchev, W. Sacks and J. Klein, Europhys.Lett. 54, p. 84 (2001).

5. K. McElroy, et al, Science 309, p. 1048 (2005).

6. D. LeBoeuf, et al, Nat. Phys. 9, 1745 (2013).

7. P. Richard, et al, Phys. Rev. B 74, p. 094512(Sep 2006).

8. C. C. Wang, et al, Phys. Rev. B 72, p. 064513(Aug 2005).

9. L. P. Gor'kov and G. B. Teitel'baum, Phys. Rev. Lett. 97, p. 247003(Dec 2006).

10. S. Ono, S. Komiya and Y. Ando, Phys. Rev. B 75, p. 024515(Jan 2007).

11. K. I. Kugel, et. al., Supercond. Sci. Technol. 22, p. 014007 (2009).

12. M. S. Laad, I. Bradaric and F. V. Kusmartsev, Phys. Rev. Lett. 100, p. 096402 (2008).

13. M. Hashimoto, et al, Nat. Phys. 10, 483 (2014).

14. A. Kaminski, T. Kondo, T. Takeuchi and G. Gu, Philosophical Magazine, 1 (2014).

15. T. J. Reber, et al, Nat Phys 8, 1 (2012).

16. J. Zhao, et al, Proceedings of the National Academy of Sciences 110, 17774 (2013).

17. M. Hashimoto,et al, Nat Mater 14, p. 37 (2015).

18. K. Fujita,et al, Science 344, 612 (2014).

19. R.-H. He,et al, Science 331, 1579 (2011).

20. I. M. Vishik,et al, Proceedings of the National Academy of Sciences 109, 18332 (2012).

21. R. A. Cooper, et al, Science 323, p. 603 (2009).

22. N. Barii, et al, Proceedings of the National Academy of Sciences 110, 12235 (2013).

23. F. V. Kusmartsev and M. Saarela, Supercond. Sci. Technol. 22, p. 014008 (2009).

24. T. Tohayama and S. Maekawa, Supercond. Sci. Technol. 13, p. R17 (2000).

25. T. Tohayama and S. Maekawa, Phys. Rev. B. 67, p. 092509 (2003).

26. C. Kim,et al, Phys. Rev. Lett. 80, p. 4245 (1998).

27. P. W. Anderson, The Theory of Superconductivity in the HTSC (Princeton, 1977).

28. F. C. Zhang and T. M. Rice, Phys. Rev. B 37, p. 3759 (1988).

29. G. Bednorz and A. Muller, Nobel lecture (Oct 1987).

30. M. Saarela and F. Kusmartsev, J. of Supercond. and Novel Magnetism 28, 1 (2015).

31. F. V. Kusmartsev, Journal de Physique IV 9, 321 (1999).

32. F. V. Kusmartsev, Phys. Rev. Lett. 84, 530 (2000).

33. F. V. Kusmartsev, Phys. Rev. Lett. 84, 5026 (2000).

34. M. Saarela and F. V. Kusmartsev, Phys. Lett. A 202, 317 (1995).

35. M. Saarela, T. Taipaleenmäki and F. V. Kusmartsev, J. Phys. A 36, p. 9223 (2003).

36. V. Apaja, S. Denk and E. Krotscheck, Phys. Rev. B 68, p. e195118 (2003).

37. F. V. Kusmartsev, A. Luther and A. Nersesyan, JETP Lett. 55, 724 (1992).

38. F. V. Kusmartsev, Phys. Lett. A 169, 108 (1992).

39. J. Zaanen, Nature 430, 512 (2004).

40. F. V. Kusmartsev and E. I. Rashba, Czech. J. Phys. 32, p. 54 (1982).

41. I. Fugol', Advances in Physics 37, 1 (1988).

42. E. Savchenko, Y. Rybalko and I. Fugol', Pis' ma Zh. Eksp. Teor. Fiz. 42, 210 (1985).

43. N. Poccia, et al, Journal of Superconductivity and Novel Magnetism 26, 2703 (2013).

44. N. Poccia, et al, Proceedings of the National Academy of Sciences 109, 15685 (2012).

45. P. Phillips, Rev. Mod. Phys. 82, 1719(May 2010).

46. Z. Viskadourakis,et al, Phys. Rev. B 85, p. 214502(Jun 2012).

47. S. Mukherjee,et al, Phys. Rev. B 85, p. 140405(Apr 2012).

48. A. Bianconi, Nature Physics 9, 536 (2013).

49. S. A. H. A. Donos, Nature Physics 9, 649 (2013). 